\title{
Cuidados de enfermagem a recém-nascido com infecção de sítio cirúrgico incisional profunda: relato de caso
}

\author{
Nursing care of a newborn with deep incisional surgical site infection: a case report \\ Cuidados de enfermería a un recién nacido con infección del sitio \\ quirúrgico incisional profunda: informe de un caso
}

Como citar este artigo:

Secco IL, Danski MTR, Pereira HP, Almeida TQR, Cruz TR. Nursing care of a newborn with deep incisional surgical site infection: a case report. Rev Esc Enferm USP. 2021;55:e03769. https://doi.org/10.1590/S1980-220X2020047203769

\author{
Izabela Linha Secco ${ }^{1}$ \\ Mitzy Tannia Reichembach Danski² \\ Higor Pacheco Pereira ${ }^{1}$ \\ Tatiana Queiroz Ribeiro de Almeida ${ }^{1}$ \\ Talita Roberta Cruz ${ }^{3}$ \\ ${ }^{1}$ Universidade Federal do Paraná, \\ Programa de Pós-Graduação em \\ Enfermagem, Curitiba, PR, Brasil. \\ ${ }^{2}$ Universidade Federal do Paraná, Departamento \\ de Enfermagem, Curitiba, PR, Brasil. \\ ${ }^{3}$ Hospital Infantil Waldemar Monastier, \\ Campo Largo, PR, Brasil.
}

\section{ABSTRACT}

Objective: To describe a case of deep incisional surgical site infection in a children's hospital in South Brazil, emphasizing the nursing care measures provided to the newborn. Method: Case study with data collection from medical record, approved by the institution and the Human Research Ethics Committee. Results: From the diagnosis of surgical site infection, a plan was established for specific care of the lesion using occlusive dressings made with technologies aimed at accelerating the second-intention healing process. Conclusion: Despite the severity of the lesions, the scientific knowledge and the skill of the nurses during treatment of the surgical site infection provided the newborn with full surgical wound healing and hospital discharge before the estimated moment.

\section{DESCRIPTORS}

Infant, Newborn; Surgical Wound Infection; Nursing Care; Occlusive Dressings; Technology; Case Reports. 


\section{INTRODUÇÃO}

A enterocolite necrosante (ECN) é a patologia mais comum que afeta o trato gastrointestinal (TGI) de recém-nascidos $(\mathrm{RN})$ prematuros e a termo, representando um risco à vida desses pacientes ${ }^{(1)}$. A ocorrência atual da ECN é, de fato, uma manifestação do grande sucesso obtido por neonatologistas em manter $\mathrm{RN}$ vivos em idades gestacionais cada vez menores ${ }^{(2)}$. Em contrapartida à sobrevida desses prematuros, a mortalidade atribuída a essa condição não mudou nas últimas cinco décadas. Tal desfecho tem sido inevitável em $50 \%$ dos RN que requerem intervenção cirúrgica ${ }^{(2)}$.

A fisiopatologia precisa da ECN ainda não foi explicada em todos os detalhes, mas as evidências sugerem-na como um processo multifatorial que inclui isquemia e lesão do intestino com subsequentes reações inflamatórias da parede intestinal $^{(1,3)}$. Para fins descritivos e para estratificação da doença, tem sido amplamente utilizado o sistema de pontuação de Bell, que avalia o grau de gravidade da doença como leve (estágio de Bell I), moderado (estágio de Bell II) ou grave (estágio de Bell III). Seu diagnóstico pode ser estabelecido por radiografia simples de abdome, que revela pneumatose intestinal (estágio II de Bell), enquanto os casos avançados apresentam pneumoperitônio (estágio III de Bell) $)^{(2)}$.

Conforme o estadiamento da doença, o tratamento é estabelecido e pode ser dividido em duas vertentes. A conservadora inclui jejum com nutrição parenteral total, drenagem gástrica, equilíbrio de fluídos, administração de analgésicos, antibióticos e catecolaminas, além de suporte ventilatório precoce. A segunda linha de tratamento, a cirúrgica, é uma indicação absoluta quando há evidência de perfuração do TGI com pneumoperitônio (estágio III de Bell). A laparotomia permite a ressecção adequada dos segmentos intestinais acometidos e, em muitos casos, pode resultar em ostomia ${ }^{(1)}$.

A despeito da mortalidade, os $\mathrm{RN}$ sobreviventes da ECN podem experimentar sérias complicações. $O$ índice de morbidade varia de 20 a $50 \%$ e é caracterizado por estenose intestinal, síndrome do intestino curto e retardo do crescimento e neurodesenvolvimento. Ademais, necessitam de maior tempo de hospitalização e estão mais suscetíveis ao óbito antes da alta ${ }^{(2)}$.

Entretanto, uma das complicações relacionadas à ECN raramente é descrita na literatura e procede da intervenção cirúrgica: a infecção de sítio cirúrgico (ISC). Na maioria dos casos, os cuidados com a ferida operatória são minimizados, potencializando o risco para ISC. Conforme os Critérios Diagnósticos de Infecção Associada à Assistência à Saúde em Neonatologia ${ }^{(4)}$, as ISC são infecções relacionadas a procedimentos cirúrgicos com ou sem colocação de prótese/ implantes em neonatos internados ou ambulatoriais, sendo classificadas conforme as camadas da pele acometidas.

Como a pele é a primeira linha de defesa contra fatores externos nocivos, sua função de barreira é perdida quando esse órgão sofre alguma lesão de continuidade. Assim, já não pode proteger as funções fisiológicas dos órgãos internos e está sujeita a infecção microbiana ${ }^{(5-6)}$. Uma vez infectada, a lesão afetará gravemente a vida e saúde do paciente ${ }^{(7)}$.
Diante da vulnerabilidade dos pacientes neonatais, são poucas as intervenções que não apresentam risco e o enfermeiro precisa saber identificar os produtos e procedimentos adequados. A integridade do tegumento deve ser a métrica para alavancar a qualidade da assistência neonatal e, na prática clínica, o enfermeiro deve atualizar o conhecimento necessário a fim de executar o cuidado baseado em evidências com o RN. Para isso, pode utilizar tecnologias que ofereçam informações adequadas e auxiliem no processo de tomada de decisão ${ }^{(8)}$.

Portanto, o objetivo deste relato de caso é descrever os principais cuidados de enfermagem em uma RN submetida à laparotomia por ECN, que evoluiu com ISC.

\section{MÉTODO}

\section{TIPO DE ESTUDO}

A metodologia adotada para o desenvolvimento deste estudo foi o relato de caso, norteado pela ferramenta Case Report Guidelines (CARE) $)^{(9)}$.

Os relatos de caso têm por objetivo a expansão local, nacional e internacional do conhecimento profissional acerca de uma manifestação clínica, abordagem diagnóstica ou terapêutica alternativa de uma doença para melhorar a qualidade dos cuidados prestados ao paciente ${ }^{(10)}$.

\section{Cenário}

O presente estudo ocorreu em um hospital infantil da região metropolitana de Curitiba, apto para atender crianças entre 0 e 18 anos, o qual possui unidades de internação cirúrgica e clínica, Unidade de Terapia Intensiva Neonatal (UTIN) e Unidade de Terapia Intensiva Pediátrica, além de Centro Cirúrgico (CC), laboratório, sala de coleta de leite materno, centro de imagem e ambulatório de especialidades médicas.

A internação da RN ocorreu em uma UTIN do tipo III que possui 20 leitos ativos, recebe pacientes de todo o estado do Paraná e conta com equipe multidisciplinar composta por enfermeiros, técnicos de enfermagem, médicos, fisioterapeutas, fonoaudiólogas, serviço social e psicologia para a assistência integral.

\section{Critérios de Seleção}

A RN em questão foi escolhida para o presente estudo por apresentar diagnósticos médicos concomitantes de ECN e ISC e estar internada na UTIN no ano de 2020.

\section{Coleta de Dados}

Os dados foram coletados do prontuário da paciente entre 20 e 30 de setembro de 2020 pelos autores que atuaram na assistência direta ao RN. Para extrair as informações de maneira sistematizada, foi utilizado um instrumento elaborado pelos pesquisadores, que abrangeu dados da $\mathrm{RN}$ desde a admissão até a alta da UTIN, dividido nos seguintes tópicos: diagnósticos médicos, história materna e da $\mathrm{RN}$, especialidades médicas (pulmonar, cardiovascular, oftalmologia, neurologia, ortopedia, otorrinolaringologia, cirurgia pediátrica), 
infecção, acesso venoso, vacinas, desenvolvimento, intercorrências e plano terapêutico.

Em relação ao registro fotográfico, os mesmos autores que realizaram a coleta de dados em prontuário capturaram as fotos mediante consentimento prévio por escrito dos responsáveis legais.

\section{ANÁLISE E TRATAMENTO DOS DADOS}

A análise dos dados ocorreu de forma descritiva por meio das fotos e informações obtidas do prontuário do paciente.

\section{Aspectos éticos}

O projeto foi aprovado pelo Comitê de Ética em Pesquisa com Seres Humanos do Complexo Hospital de Clínicas Universidade Federal do Paraná, conforme parecer consubstanciado número 3.763.468 de 12/12/2019, respeitando os preceitos éticos da Resolução n ${ }^{\circ} 466 / 2012$, do Conselho Nacional de Saúde. Foi autorizada pelo responsável legal a divulgação das imagens por meio da assinatura do termo de autorização para uso de imagem.

\section{RESULTADOS}

\section{Relato do CAso}

A mãe de 27 anos era primigesta, fez cinco consultas de pré-natal e tinha 29 semanas e 4 dias de gestação. Foi admitida no centro obstétrico em 24/04/2020 em trabalho de parto; foram administradas inibição e duas doses de corticoide. Em 28/04, a inibição foi suspensa e a mãe evoluiu para parto vaginal sem intercorrências. Possuía tipagem sanguínea $\mathrm{B}+\mathrm{e}$ VDRL e HIV negativos e era imune a toxoplasmose.

Paciente E.F.S.J., nascida em 29/04/20 às 02h40 em bom estado geral, apresentou frequência cardíaca acima de $100 \mathrm{bpm}$, tônus adequado, choro forte, Apgar 7/8, peso $1,480 \mathrm{~kg}$ e idade gestacional de 29 semanas e 5 dias; não necessitou de manobras de reanimação. Com 12 dias de vida (11/05), evoluiu com enterorragia e foi mantida em jejum; iniciou-se tratamento sistêmico com piperacilina e a paciente recebeu transfusão de hemácias. Radiografia abdominal apresentava pneumatose; contudo, sem pneumoperitônio. Em virtude da ausência de cirurgia pediátrica no hospital de origem, foi solicitada uma vaga pela Central de Leitos do Paraná à UTIN do hospital infantil e a RN foi admitida em 13/05/20 às 19 h00.

Foram estabelecidos os seguintes diagnósticos médicos durante o período de internação: RN prematura, adequada para idade gestacional, membrana hialina grau I, hipoglicemia, enterocolite, síndrome do intestino curto, hiponatremia, colestase, anemia, deiscência de ferida operatória, sepse fúngica, hemorragia peri intraventricular grau I, hipotermia e, por fim, reconstrução intestinal.

Ao exame físico na admissão, apresentava-se em regular estado geral, corada e hidratada, ativa, ictérica, escala de dor igual a 6 pela Neonatal Infant Pain Scale (NIPS). Permaneceu em ventilação mecânica, apresentando expansibilidade torácica simétrica, murmúrios vesiculares diminuídos à esquerda, mas ausência de desconforto respiratório, ausculta cardíaca normofonética, abdome distendido, tenso, doloroso à mínima palpação, ausência de peristaltismo e resíduo gástrico bilioso em média quantidade. Apresentava ainda extremidades inferiores sem edema e perfusão tissular periférica adequada. As condutas iniciais foram jejum absoluto com reposição hidroeletrolítica, coleta de exames laboratoriais e radiografia abdominal. Cateter venoso central inserido na origem encontrava-se permeável; a antibioticoterapia com piperacilina foi continuada e iniciou-se analgesia contínua com fentanil. A paciente foi avaliada pela equipe de cirurgia pediátrica (CIPE).

Em 14/05, pela manhã, foi observada piora clínica e laboratorial: acidose metabólica, hiperpotassemia e plaquetopenia. A paciente necessitou de droga vasoativa para recuperação hemodinâmica e foi realizado cateterismo vesical de demora como preparo pré-operatório, em virtude do risco iminente de cirurgia. Ecografia de abdome mostrou ascite moderada, radiografia de controle sem pneumoperitônio, mas com pobreza de ar em quadrantes abdominais. Recebeu avaliação da CIPE, que orientou conduta expectante. À tarde, exame de imagem e quadro clínico foram reavaliados pelo cirurgião e optou-se por intervenção cirúrgica. A paciente foi encaminhada ao CC às $16 \mathrm{~h} 00$ e foi realizada laparotomia exploratória, que evidenciou ECN do íleo ao cólon transverso, além de necrose desses segmentos; portanto, foi realizada enterectomia de íleo (aproximadamente $20 \mathrm{~cm}$ ) e de colón ascendente até o transverso e, na sequência, ileostomia e colostomia (Figura 1).

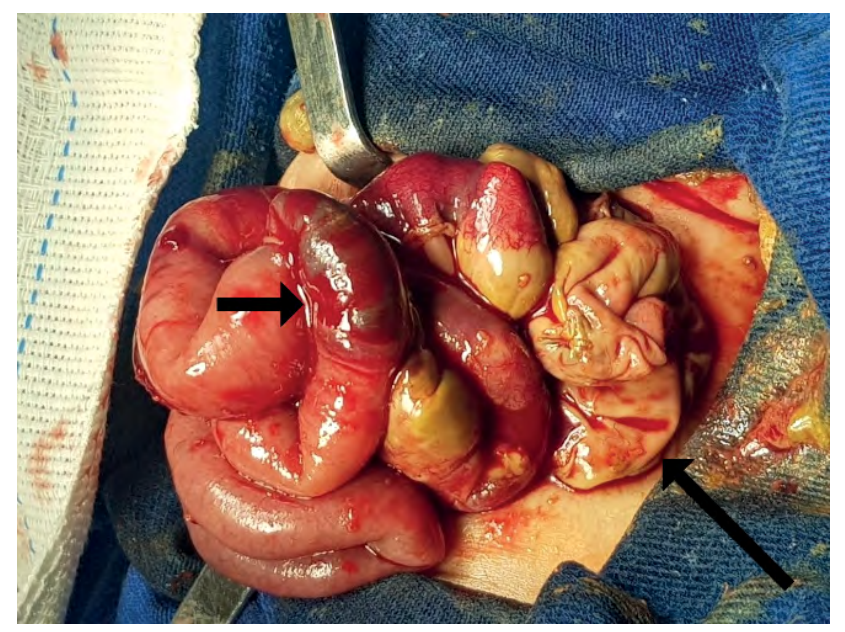

Figura 1 - Transoperatório. Acometimento intestinal extenso e evidência de necrose com perfuração (seta longa). Bolsas de ar dentro na parede intestinal resultantes da fermentação bacteriana (seta curta).

Durante o transoperatório, a paciente apresentou sangramento moderado de difícil localização e hemostasia, sendo administradas hemácias e plaquetas. Foram encaminhadas duas peças anatômicas para biópsia, que corroboraram o diagnóstico clínico de ENC. A paciente retornou à UTIN às $18 \mathrm{~h} 45$ hipotérmica $\left(35^{\circ}\right)$, acomodada em incubadora 
aquecida e umidificada, permaneceu sedada com pancurônio e fentanil e recebeu expansão volumétrica, mas hemodinamicamente estável. Associou-se metronidazol à piperacilina. O curativo oclusivo com gaze estéril e micropore em ferida operatória abdominal já estava saturado com exsudato sanguinolento. Apesar da gravidade, manteve-se estável no pós-operatório imediato (14 a 15/05).

Nos primeiros dois dias de pós-operatório (16 e 17/05), o abdome apresentava-se globoso e tenso, ruídos hidroaéreos ausentes e bordas da ferida operatória hiperemiadas e com presença de exsudato purulento em pequena quantidade; realizou-se cobertura especial com Mepitel Transfer, ao invés da gaze, conforme orientação da comissão de cuidados com a pele da instituição. No plantão noturno do dia $17 / 05$, evoluiu com piora laboratorial, sendo necessário escalonar antibioticoterapia para meropenem e vancomicina. Nos dias subsequentes, foi observado aumento gradativo dos sinais flogísticos em ileostomia com alteração da coloração do ostoma (isquemia) e drenagem de exsudado esverdeado em média quantidade. Em 20/05, a CIPE detectou deiscência de aponeurose nesse local. Por conseguinte, em 21/05 a paciente foi submetida à reabordagem cirúrgica abdominal devido à necrose de ostomia com saída de fezes e exsudado ao redor. Durante o procedimento, foram identificadas duas novas perfurações do intestino delgado próximo à ileostomia; procedeu-se com ressecção desse segmento $(3 \mathrm{~cm})$ e refez-se colostomia em região supra umbilical.

Em decorrência da segunda intervenção cirúrgica e também pelo fato de a ISC já estar presente no leito da incisão abdominal, no dia 25/05 foi observado processo inflamatório mais vigoroso em relação ao primeiro (Figura 2), culminando em grande quantidade de exsudado purulento e deiscência total com risco de evisceração (28/05) (Figura 3). Diante disso, foi prescrita sedoanalgesia contínua com fentanil e midazolam, foi realizada intubação orotraqueal devido à potencialização da sedação, e instituiu-se manuseio mínimo, a fim de otimizar a cicatrização e diminuir o risco de evisceração.

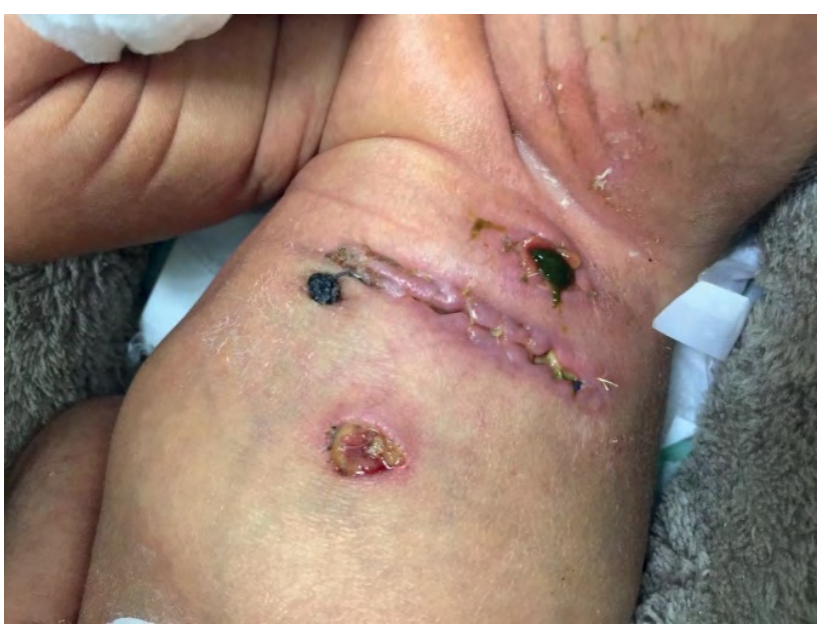

Figura 2 - Infecção de sítio cirúrgico em 25/05.

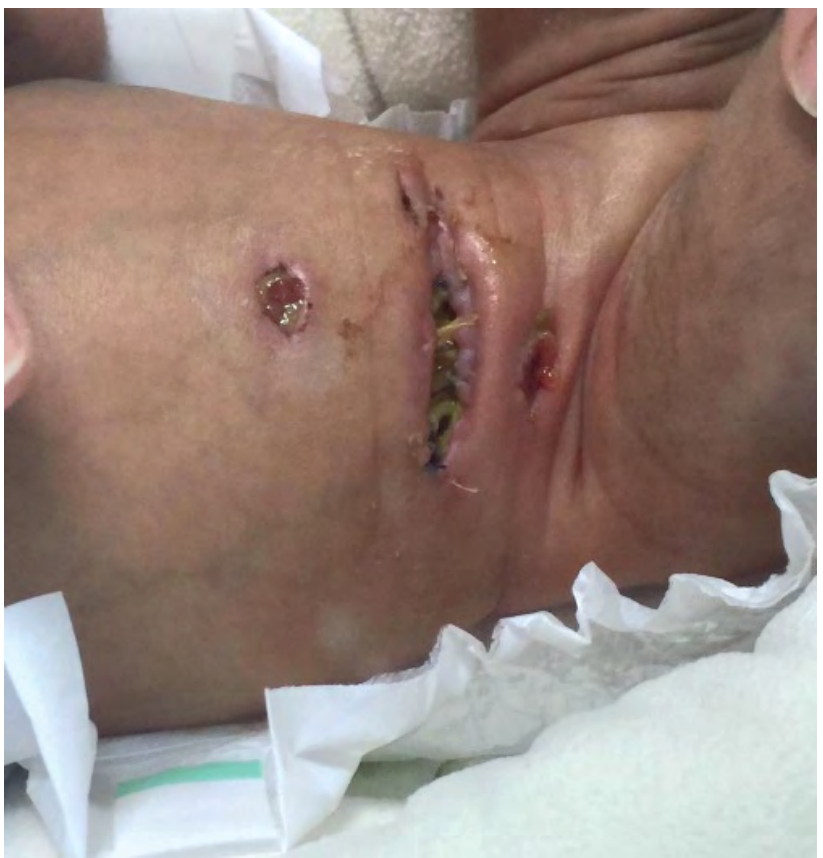

Figura 3 - Piora da ISC com deiscência total em 28/05.

A partir do dia 26/05, foi instituído tratamento tópico com curativos especiais contendo propriedades antimicrobianas (Aquacel ${ }^{\oplus}$ ) e com coberturas atraumáticas (Mepitel Transfer ${ }^{\circledR}$ ). Nesse dia, a cavidade da ferida operatória foi preenchida com Aquacel ${ }^{\circledR}$ e protegida com o Mepitel Transfer ${ }^{\circledast}$, sendo que apenas esse último material deveria ser trocado na presença de saturação. Inicialmente, foi necessário fazer 4 trocas a cada 24 horas. Progressivamente, a lesão foi diminuindo em tamanho, profundidade e exsudato, além da presença de tecido de granulação. Após cinco dias de uso (01/06), o Aquacel ${ }^{\circledR}$ foi substituído pelo hidrogel, com foco no debridamento químico da fibrina. Após a aplicação do hidrogel, era realizada cobertura secundária com gaze estéril e umidificação com soro fisiológico morno a $0,9 \%$ a cada quatro horas para potencializar a cicatrização. A cicatrização total da ferida ocorreu após 15 dias de tratamento tópico e os curativos especiais foram suspensos em 11/06.

Com a proximidade da alta hospitalar, considerando o bom quadro clínico da $\mathrm{RN}$ e as dificuldades da família frente aos cuidados específicos com os ostomas, a CIPE realizou a deileostomia em 25/06. Entretanto, após a reversão, novamente a ferida operatória evoluiu com ISC; porém, em menor gravidade. No dia $01 / 07$, a incisão apresentava pequena deiscência ao centro e média quantidade de exsudato purulento (Figura 4). Tão logo foi reiniciado o tratamento tópico com Aquacel ${ }^{\varpi}$. Dessa vez, o Mepitel Transfer ${ }^{\oplus}$ foi substituído por uma película de silicone também atraumática $\left(\right.$ Mepitel One ${ }^{\varpi}$ ), pois havia menos exsudato. A duração do tratamento dessa segunda ISC foi menor, totalizando 8 dias (09/07) (Figura 5). Todas as condutas acerca dos curativos utilizados foram estabelecidas por enfermeiros e ocorreram a beira leito. 


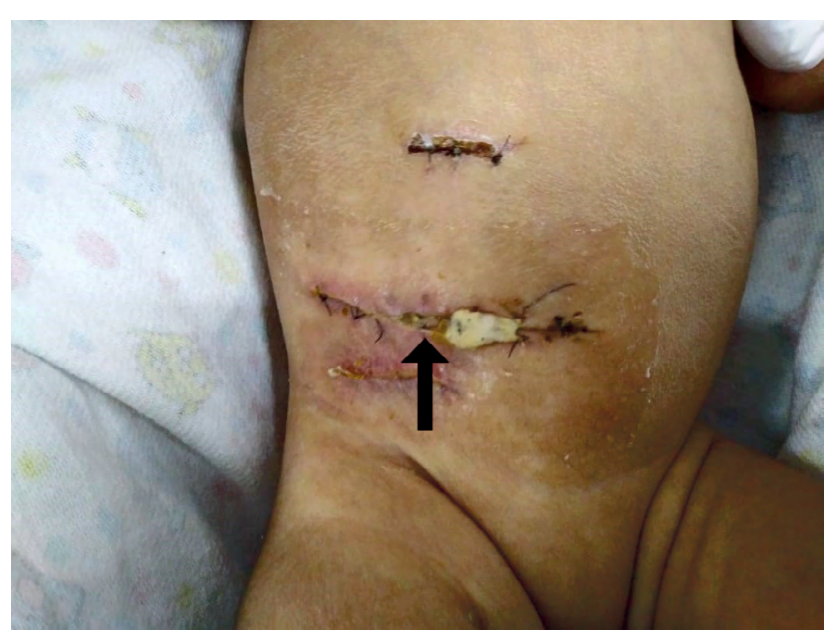

Figura 4 - Segundo episódio de ISC, apresentando pequena deiscência no centro da ferida.

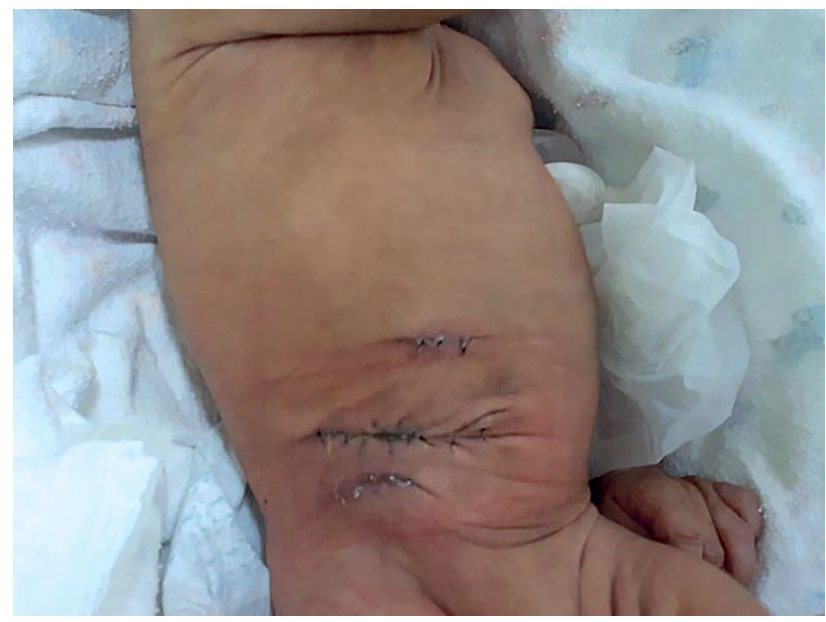

Figura 5 - Final do tratamento em 09/07.

A paciente recebeu alta da UTIN para enfermaria de lactentes em 15/07 e obteve alta hospitalar em 17/07, pesando 2,620 kg, em aleitamento materno predominante mais complemento com fórmula em mamadeira. Prescreveu-se antibioticoterapia profilática para intestino curto com bactrim, metronidazol, nistatina e vitamina B12. A paciente foi encaminhada para os ambulatórios de "bebê de risco", nutrologia e oftalmopediatria.

\section{DISCUSSÃO}

Apesar do conhecimento já construído e de diretrizes previamente estabelecidas para o manejo da ISC em adultos e, mais recentemente, em crianças, ainda pouco se sabe sobre essa condição clínica em $\mathrm{RN}$ e lactentes ${ }^{(11)}$. Diante do escasso conhecimento baseado em evidência acerca dos cuidados de enfermagem com a ISC nessa população, a prática profissional comesses tipos de lesões e a escolha de coberturas apropriadas tendem a refletir a experiência profissional do enfermeiro assistencial e suas preferências ${ }^{(12-13)}$.
Além disso, "nos últimos anos, as tecnologias para o tratamento de feridas vêm evoluindo consideravelmente, e inúmeros curativos estão sendo disponibilizados no mercado, causando, inclusive, certa confusão no momento da escolha". Esse cenário exige do enfermeiro neonatal o conhecimento das "características da pele predisposta a lesões, os mecanismos de injúria, a fisiologia da cicatrização e os fatores que a afetam”. Esse profissional "também deve saber sobre a avaliação e o tratamento de feridas, com base nas melhores evidências para restaurar a função e aparência com o mínimo de complicações"(14).

Nesse contexto, o manejo de feridas representa um desafio para a enfermagem neonatal ${ }^{(15)}$, mas é dentro dessas limitações que deve emergir o protagonismo do enfermeiro no planejamento de cuidados com a ISC. O tratamento para essas lesões, ainda que considerado interdisciplinar, é predominantemente liderado por esse profissional, que desenvolve o processo de enfermagem à beira do leito e diretamente junto ao paciente com intervenções especificas nos cuidados com feridas ${ }^{(13,15)}$.

Ao considerar a ISC como uma complicação pós-operatória, um dos cuidados de enfermagem iniciais de extrema importância inclui "a realização dos curativos nas incisões, medida importante para evitar a contaminação e proliferação de microrganismos, propiciando as condições ideais para o processo de cicatrização da ferida operatória. Os profissionais de enfermagem são responsáveis por realizar os curativos, cuja finalidade é garantir e auxiliar o tratamento da lesão estabelecida, de modo a minimizar o risco de infecção e promover ambiente favorável para que haja o processo de cicatrização. $\mathrm{O}$ enfermeiro, em especial, supervisiona esse procedimento e avalia a evolução da lesão, para que o curativo de escolha seja o mais adequado, considerando as características e o leito da ferida"(16).

"O curativo ideal modifica-se de acordo com a evolução da lesão e a escolha da cobertura a ser utilizada depende do tipo do tecido que se encontra no leito da ferida, da profundidade, da quantidade de exsudato e da presença de sinais flogísticos. Deve-se selecionar um produto que propicie um ambiente adequado para a cicatrização através da manutenção de um meio úmido. Ademais, precisa oferecer proteção contra invasão bacteriana, ser de fácil aplicação, adaptação e remoção, além de proporcionar conforto ao paciente e evitar trocas frequentes"(14).

Em lesões mais complexas, isto é, na presença de tecido desvitalizado e exsudato, são recomendadas coberturas que possuem função de debridamento autolítico e de combate à infecção. O debridamento autolítico, frequentemente usado em feridas neonatais, depende de um ambiente aquecido e úmido para atuar, o qual é apenas alcançado pelo uso de curativos especiais. Tal habilidade permite aos macrófagos digerirem o tecido necrótico e promove a granulaçãa ${ }^{(15,17)}$. O hidrogel é um composto que favorece essa ação, apresentando bons resultados na população neonatal ${ }^{(12,17)}$.

Ao escolher o curativo mais adequado, o enfermeiro também deve levar em consideração a presença de exsudato. Em sinergia ao hidrogel, curativos que contenham prata em sua composição, como o Aquacel ${ }^{\oplus}$, são indicações apropriadas para uso neonatal, pois absorvem o exsudato e ajudam a 
promover a umidade no leito da ferida, contribuindo para o debridamento autolítico ${ }^{(15)}$.

Curativos adesivos de silicone, como o Mepitel One ${ }^{\oplus}$ também são indicados em neonatologia por resultarem em menor trauma à pele do $\mathrm{RN}^{(17)}$. Quando aplicados como curativos primários, permitem que o exsudato seja transferido à cobertura secundária, reduzindo o efeito de maceração. Outra característica que os torna particularmente úteis em feridas neonatais é a sua eficácia em lesões de formato incomum em locais difíceis ${ }^{(15)}$.

Apesar da carência de publicações a respeito dos cuidados de enfermagem com ISC em neonatologia, alguns relatos de experiência corroboram a associação do hidrogel e Aquacel $^{\oplus}$ no tratamento dessas lesões. Enfermeiros neonatais relataram o uso dessas coberturas especiais em um $\mathrm{RN}$ prematuro que evoluiu com ISC após laparotomia exploratória e ileostomia. Em dez dias de tratamento tópico, a deiscência provocada pela infecção foi totalmente resolvida ${ }^{(17)}$.

Outro relato de caso publicado por uma enfermeira pediátrica abordou os cuidados de enfermagem a uma criança que desenvolveu ISC e evisceração intestinal após ileostomia. A equipe de enfermagem realizou a avaliação integral da criança, estabelecendo metas e implementando um cuidado holístico. Guiados pelas teorias da preparação do leito da ferida e da manutenção do meio úmido, um dispositivo simples de pressão negativa criado pela equipe e curativos modernos foram utilizados em diferentes estágios para gerir eficazmente o exsudato, remover a necrose e promover a granulação e a cicatrização completa da ferida. Por meio desse cuidado individualizado, a criança recebeu alta após 32 dias de internamento ${ }^{(18)}$.

Sendo assim, os cuidados de enfermagem pautados em evidência científica e nas necessidades do $\mathrm{RN}$, aliados à expertise do enfermeiro, completam a tríade da melhor prática profissional, permitindo a entrega segura de cuidados e a recuperação definitiva de $\mathrm{RN}$ criticamente enfermo em situações de risco de vida, como a ISC incisional profunda.

\section{LIMITAÇÕES DO ESTUDO}

A maioria das pesquisas envolvendo a ENC no cenário neonatal retratam complicações relacionadas à Síndrome do Intestino Curto, ao neurodesenvolvimento, septicemia e sobrevida dos $\mathrm{RN}$ acometidos por essa patologia. Entretanto, raramente reportam a ISC como complicação inerente à intervenção cirúrgica. Portanto, a limitação deste estudo é demonstrada pela escassez de publicações científicas sobre a ISC como uma complicação da ECN decorrente da necessidade de cirurgia, corroborando que a experiência vivenciada neste relato de caso é importante para auxiliar outras equipes no manejo dessa complicação.

\section{Avanços para a ÁREA DA ENFERMAGEM}

Este relato de caso propõe-se a auxiliar o profissional Enfermeiro em relação aos cuidados específicos em ISC no cenário da ENC, patologia muito frequente na UTIN. A assistência de enfermagem oferecida ao RN em questão, além de baseada em evidência, torna-se replicável em outros casos e contribui para a efetividade do cuidado.

\section{CONCLUSÃO}

Os $\mathrm{RN}$ internados em UTIN possuem inúmeros fatores de risco que potencializam lesões de pele e complicações inerentes. O objetivo deste relato de experiência foi descrever quais foram os principais cuidados de enfermagem no tratamento da ISC em um paciente neonatal. Verificou-se que a equipe de enfermagem possui um papel de protagonismo nessa prática, liderada pelo enfermeiro quando assume a responsabilidade no gerenciamento dessas lesões. Estabelecer um cuidado holístico, sistematizado e baseado em evidências conduz a equipe de enfermagem ao sucesso na abordagem terapêutica, traz satisfação profissional e, sobretudo, recuperação definitiva do $\mathrm{RN}$.

\section{RESUMO}

Objetivo: Descrever um caso de infecção de sítio cirúrgico do tipo incisional profunda ocorrido em um hospital infantil do Sul do Brasil, evidenciando os cuidados de enfermagem com o recém-nascido. Método: Estudo de caso com coleta de dados em prontuário, aprovado pela Instituição e pelo Comitê de Ética em Pesquisa com Seres Humanos. Resultados: A partir do diagnóstico de infecção de sítio cirúrgico, foi estabelecido um plano de cuidados específicos com a lesão, utilizando curativos oclusivos compostos por tecnologias especiais para acelerar o processo de cicatrização por segunda intenção. Conclusão: Apesar da gravidade das lesões, o conhecimento científico e a habilidade dos enfermeiros assistenciais no tratamento da infecção de sítio cirúrgico proporcionaram ao neonato a cicatrização completa da ferida operatória e alta hospitalar previamente ao tempo estimado.

\section{DESCRITORES}

Recém-Nascido; Infecção da Ferida Cirúrgica; Cuidados de Enfermagem; Curativos Oclusivos; Tecnologia; Relatos de Casos.

\section{RESUMEN}

Objetivo: Describir un caso de infección del sitio quirúrgico incisional profunda ocurrido en un hospital infantil del sur de Brasil, destacando los cuidados de enfermería con el recién nacido. Método: Estudio de caso con coleta de datos del expediente, aprobado por la Institución y por el Comité de Ética en Investigación con Seres Humanos. Resultados: A partir del diagnóstico de infección del sitio quirúrgico, se estableció un plan de cuidados específicos con la lesión, utilizando apósitos oclusivos compuestos por tecnologías especiales para acelerar el proceso de curación por segunda intención. Conclusión: A pesar de la gravedad de las lesiones, el conocimiento científico y la habilidad de los enfermeros en el tratamiento de la infección de sitio quirúrgico proporcionaron al neonato una cicatrización completa de la herida operatoria y alta hospitalaria antes del tiempo estimado.

\section{DESCRIPTORES}

Recién Nacido; Infección de la Herida Quirúrgica; Atención de Enfermería; Apósitos Oclusivos; Tecnología; Informes de Casos. 


\section{REFERÊNCIAS}

1. Müller MJ, Paul T, Seeliger S. Necrotizing enterocolitis in premature infants and newborns. J Neonatal Perinatal Med. 2016;9(3):233-42. https://doi.org/10.3233/NPM-16915130

2. Niño DF, Sodhi CP, Hackam DJ. Necrotizing enterocolitis: new insights into pathogenesis and mechanisms. Nat Rev Gastroenterol Hepatol. 2016;13(10):590-600. https://doi.org/10.1038/nrgastro.2016.119

3. Baranowski JR, Claud EC. Necrotizing enterocolitis and the preterm infant microbiome. Adv Exp Med Biol. 2019;1125:25-36. https://doi.org/10.1007/5584_2018_313

4. Agência Nacional de Vigilância Sanitária. Critérios diagnósticos de infecção associada à assistência à saúde: neonatologia. Brasília, DF: Agência Nacional de Vigilância Sanitária; 2017.

5. Mirani B, Pagan E, Currie B, Siddiqui MA, Hosseinzadeh R, Mostafalu P, et al. An advanced multifunctional hydrogel-based dressing for wound monitoring and drug delivery. Adv Healthc Mater. 2017;6(19):1-26. https://doi.org/10.1002/adhm.201700718

6. Zhang H, Peng M, Cheng T, Zhao P, Qiu L, Zhou J, et al. Silver nanoparticles-doped collagen-alginate antimicrobial biocomposite as potential wound dressing. J Mater Sci. 2018;53(21):14944-52. https://doi.org/10.1007/s10853-018-2710-9

7. Zhao X, Wu H, Guo B, Dong R, Qiu Y, Ma PX. Antibacterial anti-oxidant electroactive injectable hydrogel as self-healing wound dressing with hemostasis and adhesiveness for cutaneous wound healing. Biomaterials. 2017;122:34-47. https://doi.org/10.1016/j.biomaterials.2017.01.011

8. Santos SV, Ramos FR, Costa R, Batalha LM. Assessment of the quality of a software application for the prevention of skin lesions in newborns. Rev Latino-Am Enfermagem. 2020;28:e3352. https://doi.org/10.1590/1518-8345.3711.3352

9. Riley DS, Barber MS, Kienle GS, Aronson JK, Schoen-Angerer T, Tugwell P, et al. CARE guidelines for case reports: explanation and elaboration document. J Clin Epidemiol. 2017;89:218-35. https://doi.org/10.1016/j.jclinepi.2017.04.026

10. Alsaywid BS, Abdulhaq NM. Guideline on writing a case report. Urol Ann. 2019;11(2):126-31. https://doi.org/10.4103/UA.UA_177_18

11. Catania VD, Boscarelli A, Lauriti G, Morini F, Zani A. Risk factors for surgical site infection in neonates: a systematic review of the literature and meta-analysis. Front Pediatr. 2019;7(101):101. https://doi.org/10.3389/fped.2019.00101

12. King A, Stellar JJ, Blevins A, Shah KN. Dressings and products in pediatric wound care. Adv Wound Care (New Rochelle). 2014;3(4):32434. https://doi.org/10.1089/wound.2013.0477

13. Gillespie BM, Walker RM, McInnes E, Moore Z, Eskes AM, O'Connor T, et al. Preoperative and postoperative recommendations to surgical wound care interventions: a systematic meta-review of Cochrane reviews. Int J Nurs Stud. 2020;102:103486. https://doi.org/10.1016/j.ijnurstu.2019.103486

14. Santos SV, Costa R. Treatment of skin lesions in newborn children: meeting the needs of nursing staff. Rev Esc Enferm USP. 2014;48(6):985-92 https://doi.org/10.1590/S0080-623420140000700004

15. Cousins Y. Wound care considerations in neonates. Nurs Stand. 2014;28(46):61-70. https://doi.org/10.7748/ns.28.46.61.e8402

16. Vieira AL, Stocco JG, Ribeiro AC, Frantz CV. Dressings used to prevent surgical site infection in the postoperative period of cardiac surgery: integrative review. Rev Esc Enferm USP. 2018;52:e03393. https://doi.org/10.1590/s1980-220x2017011803393

17. Fox MD. Wound care in the neonatal intensive care unit. Neonatal Netw. 2011;30(5):291-303. https://doi.org/10.1891/0730-0832.30.5.291

18. Li S. Nursing of a child with wound infection and bowel evisceration following ileostomy: a case report. Ann Palliat Med. 2020;9(4):2367-74. https://doi.org/10.21037/apm-20-1189 